\title{
OP 008 THE ASSESSMENT OF HYDRATION IN ADVANCED CANCER PATIENTS USING BIOELECTRICAL IMPEDANCE ANALYSIS
}

Amara Nwosu, ${ }^{1}$ Catriona Mayland, ${ }^{1}$ Andrea Varro, ${ }^{2}$ John Ellershaw ${ }^{1} .{ }^{1}$ Marie Curie Palliative Care Institute Liverpool (MCPCIL), University of Liverpool, UK; ${ }^{2}$ Institute of Translational Medicine, University of Liverpool, UK

\subsection{6/bmjspcare-2014-000654.8}

Background Current hydration assessments methods are limited in cancer patients approaching death. Bioelectrical impedance analysis (BIA) is an accurate validated method of assessing body composition; however its clinical use in advanced cancer is uncertain.

Aims To measure hydration in advanced cancer patients using $\mathrm{BIA}$, in order to determine the relationship between symptoms, biochemistry and performance status.

Methods A cross-sectional prospective analysis of advanced cancer patients within a specialist palliative care unit in Liverpool, UK. BIA raw measurements, Resistance (R-Ohm), Reactance, (Xc-Ohm), total body water (TBW-through proxy measurement using height/resistance $\left.\left[\mathrm{Ht}^{2} / \mathrm{R}\right]\right)$, Phase angle (PA) and BIA vector analysis (BIVA) were used to compare for differences in hydration based on WHO performance status $(0=$ asymptomatic, $4=$ bedbound), symptoms, physical examination and biochemistry.

Findings From a possible 97 patients, 78 (80\%) provided consent to participate. $\mathrm{Ht}^{2} / \mathrm{R}$ was lower in patients with a WHO performance status of 4 compared to those with a performance status $<4$ (mean 42.1 [SD 9.0] vs. 52.3 [14.6], $\mathrm{p}=0.002$ ), and greater in those with clinically detectable oedema (mean 57 [SD 17.4] vs. 47.7 [12.4], $\mathrm{p}=0.027$ ). $\mathrm{Ht}^{2} / \mathrm{R}$ correlated negatively with thirst $[\mathrm{r}=-0.31, \mathrm{p}=0.006]$, dry mouth $[\mathrm{r}=-0.294, \mathrm{p}=0.009]$ and fatigue $[\mathrm{r}=-0.285$, $\mathrm{p}=0.011$ ). BIVA showed significant difference in hydration between groups classified by the presence or absence of oedema $(p=0.03)$ and dry mouth $(p=0.04)$. There was no significant relationship between hydration $\left(\mathrm{Ht}^{2} / \mathrm{R}\right.$ and BIVA) vs. biochemical tests or between symptoms vs. biochemistry or performance status.

Conclusions In advanced cancer, hydration (as measured by $\mathrm{Ht}^{2} / \mathrm{R}$ and BIVA) relates to clinically measurable signs and symptoms. Further work is needed to determine whether BIA can be used to guide the management of fluid states in advanced cancer. 